\title{
Delay-Coordinates Embeddings as a Data Mining Tool for Denoising Speech Signals.
}

\author{
D. Napoletani ${ }^{1}$, C.A. Berenstein ${ }^{2}$, T. Sauer ${ }^{3 a}$, D.C. Struppa ${ }^{3 b}$ and D. Walnut ${ }^{3 c}$.
}

\begin{abstract}
In this paper we utilize techniques from the theory of non-linear dynamical systems to define a notion of embedding threshold estimators. More specifically we use delay-coordinates embeddings of sets of coefficients of the measured signal (in some chosen frame) as a data mining tool to separate structures that are likely to be generated by signals belonging to some predetermined data set. We describe a particular variation of the embedding threshold estimator implemented in a windowed Fourier frame, and we apply it to speech signals heavily corrupted with the addition of several types of white noise. Our experimental work seems to suggest that, after training on the data sets of interest, these estimators perform well for a variety of white noise processes and noise intensity levels. The method is compared, for the case of Gaussian white noise, to a block thresholding estimator.
\end{abstract}

Index Terms-Threshold estimators, delay-coordinates embeddings, nonlinear systems, data-driven denoising.

\section{INTRODUCTION}

In this paper we explore the performance of a method of denoising that is designed to be efficient for a variety of white noise contaminations and noise intensities, while keeping a fixed choice of parameters of the algorithm itself (adapted to the class of signals to denoise). The method is based on a loose distinction between the geometry of delay-coordinates embeddings of, respectively, deterministic time series and non-deterministic ones. Delay-coordinates embeddings are the basis of many applications of the theory of non-linear dynamical systems, see for example [ASY] or [KS], our work stands apart from previous applications of embeddings in that no exact modelization of the underlyning signals (through the delay-coordinates embeddings) is needed nor attempted here. Instead, we measure the overall 'squeezing' of the dynamics along the principal direction of the embedding image by computing the quotient of the largest and smallest singular values.

We define first of all the context in which we look for signal estimators. Let $F[n], n=1, \ldots, N$, be a discrete signal of length $N$, and let $X[n]=F[n]+W[n], n=1, \ldots, N$, be a contaminated measurement of $F[n]$, where $W[n]$ are realizations of a white noise process $W$, throughout this paper we use the notation $E(*)$ to denote the expected value

${ }^{1}$ School of Computational Sciences, George Mason University, Fairfax, VA 22030, email:dnapolet@gmu.edu

2 Institute for Systems Research, University of Maryland, College Park, MD 20742,email:carlos@glue.umd.edu

$3 a, b, c$ Department of Mathematical Sciences,George Mason University,Fairfax, VA 22030,emails: ${ }^{3 a}$ tsauer@gmu.edu; ${ }^{3 b}$ dstruppa@gmu.edu; $3 c$ dwalnut@gmu.edu of a quantity $*$.

Generally we are interested in estimators $F$ such that the expected mean square error $E\left\{|f-F|^{2}\right\}$ is as small as possible. For a given discrete orthonormal basis $B=\left\{g_{m}\right\}$ of the $N$ dimensional space of discrete signals, we can write: $X=\sum_{m=0}^{N-1} X_{B}[m] g_{m}$ where $X_{B}[m]=<X, g_{m}>$ is the inner product of $X$ and $g_{m}$. Given such notation, we can define a class of estimators that is amenable to theoretical analysis, namely the class of diagonal estimators of the form $\tilde{F}=\sum_{m=0}^{N-1} d_{m}\left(X_{B}[m]\right) g_{m}$ where $d_{m}\left(X_{B}[m]\right)$ is a function that depends only on the value of $X_{B}[m]$. One particular kind of diagonal estimator is the hard thresholding estimator $\tilde{F}_{T}$ (for $T$ some positive real number) defined by the choice

$$
\tilde{F}_{T}=\sum_{m=0}^{N-1} d_{m}\left(X_{B}[m]\right) g_{m}
$$

where

$$
d_{m}\left(X_{B}[m]\right)=X_{B}[m] \text { if }\left|X_{B}[m]\right|>T
$$

and

$$
d_{m}\left(X_{B}[m]\right)=0 \text { otherwise. }
$$

If $W[n]$ are realizations of a white normal distribution with variance $\sigma^{2}$, then it is shown in [DJ] that $\tilde{F}_{T}$, with $T=\sigma \sqrt{2 \log N}$, achieves almost minimax risk (when implemented in a wavelet basis) for the class of signals $f[n]$ of bounded variation. The possibility of proving such a striking result is based, in part, on the fact that the coefficients $W_{B}[n]$ are realizations of a Gaussian white noise process in any basis $B$.

Several techniques have been developed to deal with the non-Gaussian case, some of the most successful are the Efromovich-Pinsker (EP) estimator (see for example [ELPT] and references threin) and the block threshold estimators of Cai and collaborators (see [CS],[C] and the more recent $[C L])$. In these methods, the variance of the white process needs to be estimated from the data, moreover, since the threshold is designed to evaluate intensities (or relative intensities) of the coefficients in blocks of multiwavelets, low intensity details may be filtered out as it is the case for simpler denoising methods (see also remark 3 on the issue of low intensity non-noisy features).

The method we describe in this paper does not need the knowledge of the noise intensity level (thanks to the use 
of quotients of singular values), and it is remarkably robust to changes in the type of noise distribution.

This strenght is achieved at a price, the inner parameters of the algorithm need to be adjusted to the data, this is true to some extent for the EP and block thresholding algorithms as well (see again [ELPT] and [CL]), but the number and type of parameters that need to be trained in our approach is increased by the need of choosing a 'good' delay-coordinates embedding suitable for the data we would like to denoise.

In section $\mathrm{V}$ we will explore possible ways to make the training on the data automatic, but it is yet to be seen at this stage which data sets are amenable to the analysis we propose. This paper is meant as a mostly experimental analysis that suggest the method is sound at least for one choice of data sets (namely, speech signals).

Because of the choice of applying our algorithm to a database of speech signals, we decided to use windowed Fourier frames as a basic analytical tool. This is an obvious way in which we are already adapting to the data, but more general frames $\mathcal{D}$ could be used, or even collection of frames and bases, therefore we prefer to label $\mathcal{D}$ as a dictionary of analysis.

Note that any discrete periodic signal $X[n], n \in \mathbb{Z}$ with period $N$ can be represented in a discrete windowed Fourier frame. The atoms in this frame are of the form

$$
g_{m, l}[n]=g[n-m] \exp \left(-\frac{i 2 \pi l n}{N}\right), \quad n \in \mathbb{Z} .
$$

We choose the window $g$ to be a symmetric $N$-periodic function of norm 1 and support $q$. Specifically we can choose $g$ to be the characteristic function of the $[0,1]$ interval; we realize that this may not be the most robust choice in many cases, but we have deliberately selected this function to avoid excessive smoothing which was found to adversely affect our algorithm.

Under the previous conditions $x$ can be completely reconstructed from the inner products $\mathcal{F} X[m, l]=<$ $X, g_{m, l}>$, i.e.,

$$
X=\frac{1}{N} \sum_{m=0}^{N-1} \sum_{l=0}^{N-1} \mathcal{F} X[m, l] \tilde{g}_{m, l}
$$

where

$$
\tilde{g}_{m, l}[n]=g[n-m] \exp \left(\frac{i 2 \pi l n}{N}\right), \quad n \in \mathbb{Z}
$$

We denote the collection $\left\{\left\langle X, g_{m, l}\right\rangle\right\}$ by $\mathcal{F} X$. For finite discrete signals of length $N$ the reconstruction has boundary errors. However, the region affected by such boundary effects is limited by the size $q$ of the support of $g$ and we can therefore have perfect reconstruction if we first extend $X$ suitably at the boundaries of its support and then compute the inner products $\mathcal{F} X$. More details can be found in [S] and references therein.

Since for speech signals much of the structure in the time frequency domain is contained in localized 'ridges' that are oriented in time direction, the collection $C_{p}$ of double-indexed paths

$$
\gamma_{\bar{m}, \bar{l}}=\left\{g_{m, l} \text { such that } l=\bar{l}, \bar{m} \leq m \leq \bar{m}+p\right\},
$$

where $p$ is some positive integer, will be relatively sensitive to local time changes of such ridges, since each path is a short line in the time frequency domain oriented in the time direction.

The choice of $p$ is very important as different structure in speech signals (our main case study) is evident at different time scales. Let $I=I\left(\gamma_{\bar{m}, \bar{l}}\right)=I\left(\mathcal{F} X_{\gamma_{\bar{m}, \bar{l}}}\right)$ be a function defined for each path $\gamma_{\bar{m}, \bar{l}} \in \mathcal{C}_{p}$. We define now a semi-local thresholding estimator in the window Fourier frame as follows:

$$
\tilde{F}=\frac{1}{N} \sum_{m=0}^{N-1} \sum_{l=0}^{N-1} d_{I, T}(\mathcal{F} X[m, l]) \tilde{g}_{m, l}
$$

where $d_{I, T}(\mathcal{F} X[m, l])=\mathcal{F} X[m, l]$ if $I\left(\mathcal{F} X_{\gamma_{\bar{m}, \bar{l}}}\right) \geq T$ for some $\gamma_{\bar{m}, \bar{l}}$ containing $(m, l)$, and $d_{I, T}(\mathcal{F} X[m, l])=0$ if $I\left(\mathcal{F} X_{\gamma_{\bar{m}, \bar{l}}}\right)<T$ for all $\gamma_{\bar{m}, \bar{l}}$ containing $(m, l)$.

Note that this threshold estimator is build to mirror the diagonal estimators in (1), but that the 'semilocal' quality of $\tilde{F}$ is evident from the fact that all coefficients in several $\mathcal{F} X_{\gamma}$ are used to decide the action of the thresholding on each coefficient. This procedure is similar to block thresholding estimators, with the additional flexibility of choosing the index function $I$. We propose in the next section a novel use of embedding techniques from non-linear dynamical systems theory to choose a specific form for $I$. We find in this way a variance independent estimator that does not depend significantly on the probability distribution of the random variable $W$ and such that we can adapt to the data in a flexible way.

\section{Delay-Coordinates Embedding Images of Time SERIES}

We first recall a fundamental result about reconstruction of the state space realization of a dynamical system from its time series measurements. Suppose $S$ is a dynamical system, with state space $\mathbb{R}^{k}$ and let $h: \mathbb{R}^{k} \rightarrow \mathbb{R}$ be a measurement, i.e., a continuous function of the state variables. Define moreover a function $F$ of the state variables $X$ as

$$
F(X)=\left[h(X), h\left(S_{-\tau}(X)\right), \ldots, h\left(S_{-(d-1) \tau}(X)\right)\right]
$$

where by $S_{-j \tau}(X)$ we denote the state of the system with initial condition $X$ at $j \tau$ time units earlier.

We say that $A \subset \mathbb{R}^{k}$ is an invariant set with respect to $S$ if $X \in A$ implies $S_{t}(X) \in A$ for all $t$. Then the following theorem is true (see [ASY], [SYC] and $[\mathrm{KS}]$ ):

Theorem: Let $A$ be an m-dimensional submanifold of 
$\mathbb{R}^{k}$ which is invariant under the dynamical system $S$. If $d>2 m$, then for generic measuring functions $h$ and generic delays $\tau$, the function $F$ defined in (7) is one-to-one on $A$.

Keeping in mind that generally the most significant information about $g$ is the knowledge of the attractive invariant subsets, we can say that delay maps allow to have a faithful description of the underlining finite dimensional dynamics, if any. The previous theorem can be extended to invariant sets $A$ that are not topological manifolds; in that case more sophisticated notions of dimension are used (see [SYC]).

Generally the identification of the 'best' $\tau$ and $d$ that allows for a faithful representation of the invariant subset is considered very important in practical applications (as discussed in depth in $[\mathrm{KS}]$ ), as it allows to make transparent the properties of the invariant set itself, more particularly we want to deduce from the data itself the dimension $m$ of the invariant set (if any) so that we can choose a $d$ that is large enough for the theorem to apply. Moreover the size of $\tau$ has to be large enough to resolve the image far from the diagonal, but small enough to avoid decorrelation of the delay coordinates point.

We apply the structure of the embedding in such a way that the identification of the most suitable $\tau$ and $d$ is not so crucial , even though we will see that we do need to train such parameters on the available data, but in a much simpler and straightforward way. The technical reason for such robustness in the choice of parameters will be clarified later on, but essentially we use time delay embeddings as data mining tools rather than modelization tools as usually is the case.

To understand how such data mining is possible, we start by applying the delay-coordinate procedure to the time series $W[n], n=1, \ldots, N$, for $W$ an uncorrelated random process; let the measuring function $h$ be the identity function and assume from now on that $\tau$ is an integer delay so that $F(W[n])=[W[n], W[n-\tau], \ldots, W[n-(d-1) \tau]]$. For any embedding dimension $d$, the state space will be filled according to a spherically symmetric probability distribution. Let now $\bar{Z}=\{F(Z[n]), n=1, \ldots, N\}$ be the embedding image in $\mathbb{R}^{d}$ of a time series $Z$ for any given time delay $\tau$. Then we have the following very simple, but fertile lemma that relates spherical distributions to their associated to principal directions

Lemma 1: Let $\sigma_{1}, \sigma_{d}$ be the variance of $\bar{W}$ along the first principal direction (of largest extent) and the last one (smallest) respectively. Then the expected value $E\left\{\frac{\sigma_{1}}{\sigma_{d}}\right\}$ converges to 1 as $N$ goes to infinity.

Proof: Because $W$ is a white noise process, each coordinate of $F(W[n])$ is a realization of a same random variable with some given probability density function $g$, therefore $\bar{W}$ is a realization of a multivariate random variable of dimension $d$ and symmetric probability distribution. If the expected value of $\frac{\sigma_{1}}{\sigma_{d}}=Q>1$, then a point at a distance from the origin of $\sigma_{1}$ has a greater probability to lie along the principal direction associated to $\sigma_{1}$ contradicting the fact that the probability distribution of $\bar{W}$ was symmetric.

Remark 1: Even when $X$ is a pure white noise process, the windowed Fourier frame will enforce a certain degree of smoothness along each path $\gamma$ since consecutive points in $\gamma$ are inner products of frame atoms with partially overlapping segments of $X$. So there will be some correlation in $\mathcal{F} X_{\gamma}$ even when $X$ is an uncorrelated time series, therefore it is possible in general that $I\left(\mathcal{F} X_{\gamma}\right)>>1$ even when $X$ is a white noise process.

Remark 2: Similarly, the length $p$ of $\gamma$ cannot be chosen very large in practice, while $E\left(\frac{\sigma_{1}}{\sigma_{d}}\right)$ converges to 1 for any uncorrelated processes only asymptotically for very long time series and again for small length $p$ we may have $E\left(\frac{\sigma_{1}}{\sigma_{d}}\right)>>1$.

Even with the limitations explained in the previous two remarks, it is still meaningful to set $I\left(X_{\gamma}\right)=I^{\text {svd }}\left(X_{\gamma}\right)=\frac{\sigma_{1}}{\sigma_{d}}$, and therefore we define an embedding threshold estimator to be a semilocal estimator $\tilde{F}$ (as in (2)) with the choice of index $I=I^{\text {svd }}$, what we call an embedding index. The question is now to find a specific choice of $T \geq 1$, given a choice of $\left(\mathcal{D}, C_{p}, d, \tau\right)$, that allows to discriminate a given data set (speech signals in this paper) from white noise processes.

We need therefore to study the value distribution of $I^{\text {svd }}$ for our specific choice of $\mathcal{C}_{p}$ and $\mathcal{D}$, and assuming $X$ is either an uncorrelated random process or a signal belonging to our class of speech signals.

In the next section we explore numerically this issue for the windowed Fourier frames and the collection of paths $C_{p}$ in (5).

\section{Embedding Index of SpeEch Signals AND RANDOM PROCESSES}

For a given times series $X$ and choice of parameters $(p, \tau, d)$ we can compute the collection of embedding indexes $I^{s v d}(\mathcal{F} X)=\left\{I^{s v d}\left(\mathcal{F} X_{\gamma}\right), \gamma \in C_{p}\right\}$, Define now the index cumulative function as

$$
Q_{X}(t)=\frac{\#\left\{\gamma \text { such that } I^{s v d}\left(\mathcal{F} X_{\gamma}\right)>t\right\}}{\#\{\gamma\}},
$$

i.e. for a given $t, Q_{X}(t)$ is the fraction of paths that have index above $t$.

A simple property of $Q_{X}$ will be crucial in the following discussion:

Lemma 2: If $X$ is a white noise process and $X^{\prime}=a X$ is another random process that component by component is a rescaling of $X$ by a positive number $a$, then the expected function $Q_{X}$ and $Q_{X^{\prime}}$ are equal.

Proof: Each set of embedding points generated by one specific path $\gamma$ is, coordinate by coordinate, a linear combination 
of some set of points in the original time series. Therefore if $X^{\prime}=a X, \mathcal{F} \bar{X}_{\gamma}^{\prime}=a \mathcal{F} \bar{X}_{\gamma}$, but the quotient of singular values of a set of points is not affected by rescaling of all coordinates, therefore the distributions of $I^{s v d}(\mathcal{F} X)$ and $I^{s v d}\left(\mathcal{F} X^{\prime}\right)$ are equal, but $Q_{X^{\prime}}$ and $Q_{X}$ are defined in terms of $I^{\text {svd }}$ so they are equal as well.

Remark 3: We see the use of embedding index as a possible generalization of methods like the coherent structures extraction of $[\mathrm{M}]$ section 10.5 (more details can be found in [DMA]), where it is explored the notion of correlation of a signal $X$ of length $N$ with a basis $B$, defined as

$$
\mathcal{C}(X)=\frac{\sup _{0 \leq m<N}\left|X_{B}[m]\right|}{|X|} .
$$

It turns out that in the limit $N \rightarrow \infty$ the correlation of any Gaussian white process converges to

$$
\mathcal{C}_{N}=\frac{\sqrt{2 \log _{e} N}}{\sqrt{N}}
$$

independently of the specific variance and therefore estimation of a signal $X$ is performed by retaining a coefficient $X_{B}[m]$ if $\frac{\left|X_{B}[m]\right|}{|X|}>\mathcal{C}_{N}$. In this paper the embedding index determines the coherence of a coefficient with respect to a neighbourhood of the signal and it is independent of the variance of the noise process as well.

Remark 4: As we said in section II, the choice of $p$ in $C_{p}$ is very important in practice. The speech signals that we consider are sampled at a sampling frequency of about $8100 \mathrm{pt} / \mathrm{s}$, we choose supprt of the window $q=64$ and length of the paths $p=2^{8}$, since these values seem to assure that each path will be significantly shorter than most stationary vocal emissions, a point to take into consideration when we gauge the relevance of our results.

Given this lenght $p$ for $\gamma$, we have some significant restrictions on the maximum embedding dimension $d$ and time delay $\tau$ that we can choose if we want to have for each path a sufficiently large number of points in the embedding image to be statistically significant, which we can obtain if $p>>d \tau$. Because of these restrictions we choose $d=4$ and $\tau=4$ that give $d \tau=2^{4}<<p=2^{8}$, we generate in this way 240 points for each path. We heuristically tried to adjust the embedding parameters $d$ and $\tau$ and the lenght $p$ of the paths so that the qualitative behaviour of speech signals and white noise processes was as distinct as possible, see the discussion in section IV for a possible way to make the choice of parameters automatic.

We now expand some uncorrelated zero mean random processes of length $N=2^{11}$ on the windowed Fourier frame with the set values $q=64, p=2^{8}, d=4$ and $\tau=8$. And we compute the embedding index $Q_{X}$.

The specific random processes we use here are time series with each point a realization of a random variables with:
1) Gaussian probability density function.

2) Uniform probability density function.

3) Tukey probability density function, that is, a sum of two normal distributions with uneven weight (used in [ELPT] as well), each point of the time series is a realization of the random variable $W=R N_{1}+(1-R) 4 N_{2} / \sqrt{r+16(1-r)}$, where $N_{1}$ and $N_{2}$ are Gaussian random variables, and $R$ is a Bernoulli random variable with $P(R=1)=0.9$ and $r=P(R=1)$.

4)discrete uniform pdf with values in $\{-Q, Q\}$ for some positive $Q$.

All probability density functions are set to have mean zero. and variance 1 , since by Lemma 2 we know $Q_{*}$ will not be affected by changes of the variance. One of the pdf has heavy tail (Tukey pdf) and one of them is discrete (discrete uniform pdf). The kurtosis is respectively from pdf in 1) to pdf in 4): 3 , about 1.8 , about 13 , and about 1.2

In Figure 1a we plot $Q_{X}(t)$ for the white noise processes generated with pdfs in 1)-4), averaged over 10 repetitions for each random distribution.

Remark 5: To speed up the computation, we sampled the indexes $(\bar{m}, \bar{l})$ of the paths in (5), more particularly we selected a sampling length of $S_{\bar{m}}=1$ for the frequency index $\bar{m}$ and a sampling length of $S_{\bar{l}}=p$ for the time index.

Note that the qualitative behaviour of $Q_{X}$ is very similar for all chosen distributions, in particular they all exhibit a very fast decay for larger values of $t$. The maximum $L_{2}$ distance between any two $Q_{X}$ in the interval $[0,40]$ is $\approx 0.54$ (or some $6 \%$ of the average $L_{2}$ norm of the $Q_{X}$ ), we found that even for distribution with kurtosis up to 50 the maximum distance was less that 0.8 (about $8.5 \%$ of the average $L_{2}$ norm of $Q_{X}$ ), irrispective of the specific pdf, moreover most of the error is concentrated in regions of high intensity of the derivative and it does not affect much the behaviour of the right tail of the curves $Q_{X}$.

Therefore it seems that, for our choice of $\mathcal{D}$ and $C_{p}$, reasonably heavy tail distributions will not exibit a significantly different behaviour in $Q_{X}$ with respect to the Gaussian distribution, supporting our claim that $Q_{X}$ is robust with respect to the choice of white noise distribution.

For each probability density function, the shape of $Q_{X}$ is affected by the correlation introduced by the length of $q$ (the window support of the windowed Fourier Frame): if $\tau<q$ some coordinates in each embedding point will be correlated and this will cause the decay of $Q_{X}$ to be slower when $\tau$ is smaller.

When $Q_{X}$ is computed (with the same choice of parameters) for a collection of 10 randomly selected segments of speech signals of length $2^{11}$, the rate of decay of the functions $Q_{X}$ is significantly different, and the tail of the functions is still considerably thick by the time the rate of decay of $Q_{X}$ for most random processes is almost zero (see Figure 1b). 

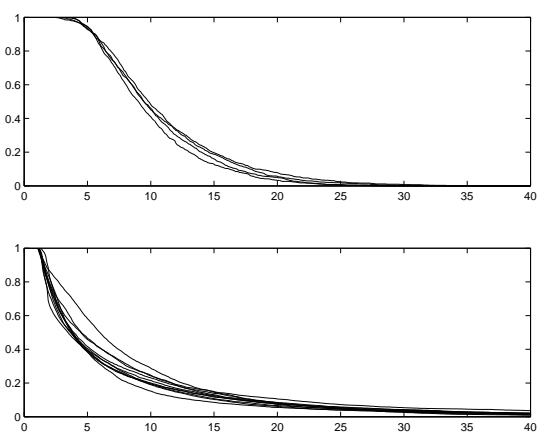

Fig. 1. From top to bottom, this figure shows $Q_{*}$, as defined in equation (7) for: a) uncorrelated random processes 1)to 4); b) ten randomly selected segment of speech signal from the TIMIT database.

Since we want to have a significantly larger fraction of paths retained for speech signals rather than noise, we can select the threshold $T$ in the following way:

(A) Determination of Threshold Given a choice of parameters $\left(\mathcal{D}, \mathcal{C}_{p}, p, \tau, d\right)$, a collection of training speech time series $\left\{S_{j}\right\}$, and a selection of white noise processes $\left\{W_{i}\right\}$, choose $T_{0}$ to be the smallest $t$ so that the mean of $Q_{S_{j}}\left(T_{0}\right)$ is one order of magnitute (10 times) larger than the mean of $Q_{W_{i}}\left(T_{0}\right)$.

This heuristic rule gives, for the parameters in this section, $T_{0} \approx 28.2$. (A) gives us as experimental way to determine a threshold $T=T_{0}$ for the index $I^{\text {svd }}$ that removes most of the time frequency structure of some predetermined noise distributions, while it preserves a larger fraction of the time frequency structure of speech signals. Since moreover 'reasonable' distributions exibited a $Q_{X}$ similar to the one of Gaussian distributions, we can in practice train the threshold only on Gaussian noise and be assured that it will be a meaningful value for a larger class of distributions.

Note that even very low energy paths could have in principle high embedding index, still, the energy concentration in paths that have very high index tends to be large for speech signals, to see that, for a given signal $X$, let

$$
E_{X}(t)=\frac{\sum\left\{\left|\mathcal{F} X_{\gamma}\right|_{2} \text { such that } I^{\text {svd }}\left(\mathcal{F} X_{\gamma}\right)>t\right\}}{\sum\left|\mathcal{F} X_{\gamma}\right|_{2}},
$$

be the fraction of the total energy contained in paths with index above $x$. We can see in Figure 2 that the amount of energy contained in paths with high index value is significantly larger for speech signals than for noise distributions.

More particularly, the fraction of the total energy of the paths carried by paths with $I^{\text {svd }}>T_{0}$ is on average 0.005 for the noise distributions and 0.15 for the speech signals, or an increase by a factor of 30 .

It seems therefore that $I^{s v d}$, with our specific choice of parameters, is quite effective in separating a subset of paths that are likely to be generated by speech signals, note
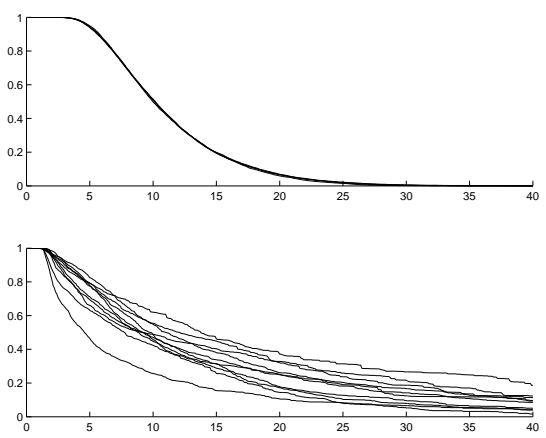

Fig. 2. From top to bottom, this figure shows $E_{*}$, as defined in equation (8) for: a) the uncorrelated random processes in Figure 1a; b) the segments of speech signals in Figure $1 \mathrm{~b}$.

moreover that similar results can be obtained with local changes of $p, \tau$ and $d$, which suggests an intrinsic robustness of the separation with respect of the parameters.

This separation ability could be due, in principle, only to the very nice properties of speech signals. Note that if, for some $\mathcal{F} X_{\gamma}, I^{\text {svd }}=\infty$, then the state realization of the time series $\mathcal{F} X_{\gamma}$ is embedded in a subspace of $R^{d}$ and therefore each point of $\mathcal{F} X_{\gamma}$ must be described as a linear function of the delay coordinates. This condition is very restrictive on the dynamics of $\mathcal{F} X_{\gamma}$, but vocal emissions are locally periodic signals, and so they do fall, at least locally, into the class of linearly predictable discrete models, i.e., processes for which $X_{k}=r\left(X_{k-1}, \ldots, X_{k-d}\right)$ for some linear function $r$ and for some integer $d$.

The complexity of these linear models increases with increasing values of the embedding dimension $d$. But this is not fully satisfactory as we would like to be able to use the embedding index $I^{\text {svd }}$ to denoise more complex dynamics that cannot be described by simple linear predictive models. Moreover for small $\tau$ we are measuring in many cases smoothness of the path and local correlation with the embedding index, yet, if we try to choose $\tau$ as large as possible with still a clear separation of the training sets, we can see differences that are not accounted for by local correlation, indeed the embedding image is squeezed along the diagonal for paths with high local smoothness, but in principle for complex dynamics the principal direction could be oriented in any direction and therefore the embedding index is much more than simply a measure of local smoothness.

There is a large literature on possible ways to distinguish complex dynamical systems from random behaviour (see for example the articles collected in [Me]), as we underlined in the previous section, much of this work stresses the identification of the proper embedding parameters $\tau$ and $d$; the contribution of this paper to this ongoing discussion is the use of embedding techniques in the context of computational harmonic analysis. This context frees us from the need to use embedding techniques to find an effective modelization of the signals, such 'blind' use of the embedding theorem is, we believe, fertile from a practical point of view, as well as a theoretical one. 
Note in any case that if the dimension of the invariant set $A$ is $d_{A}=0$, then for any white noise process $W, X+W$ has spherically symmetric embedding image and $\frac{\sigma_{1}}{\sigma_{d}} \approx 1$ for any embedding dimension $d$ as in the case of pure white noise. This means that an estimator based on $I^{\text {svd }}$ is not able to estimate noisy constant time series on a given path $\gamma$. This restriction can be eased by allowing information on the distance of the center of the embedding image to be included in the definition of the embedding threshold estimator. In this paper for simplicity we assumed $d_{A}>0$ for all paths in $C_{p}$. That seems to be sufficient in analyzing speech signals.

\section{ATtENUATED EMBEDDING Estimators}

In this section we develop an algorithm based on these ideas. The notion of semilocal estimator is slightly expanded to improve the actual performance of the estimator itself. To this extent, define tubular neighborhoods for each atom in the windowed Fourier frame, i.e.:

$$
\mathcal{O}\left(g_{m, l}\right)=\left\{g_{m^{\prime}, l^{\prime}} \text { s.t. }\left|l^{\prime}-l\right| \leq 1,\left|m^{\prime}-m\right| \leq 1\right\},
$$

Such neighborhoods are used in the algorithm as a way to make a decision on the value of the coefficients in a two dimensional neighborhood of $\mathcal{F} X_{\gamma}$ based on the the analysis of the one dimensional time series $\mathcal{F} X_{\gamma}$ itself.

(C1) Set $\tilde{F}=0$.

(C2) Given $X$, choose $q>0$ and expand $X$ in a windowed Fourier frame with window size $q$.

(C3) Choose sampling intervals $S_{\bar{l}}$ for time coordinate and $S_{\bar{m}}$ for the frequency coordinate. Choose the path length p. Build a collection of paths $\mathcal{C}_{p}$ as in (5).

(C4) Choose embedding dimension $d$ and delay $\tau$ along the path. Compute the index $I^{\text {svd }}\left(\mathcal{F} X_{\gamma_{\bar{m}, \bar{l}}}\right)$ for each $\mathcal{F} X_{\gamma_{\bar{m}, \bar{l}}} \in \mathcal{C}_{p}$. Use (A) to find the threshold level $T$.

(C5) Choose attenuation coefficient $\alpha$. Set $\mathcal{F} Y[m, l]=$ $\alpha \mathcal{F} X[m, l]$ if $I^{s v d}\left(\mathcal{F} X_{\gamma}\right) \geq T$ for some $\gamma$ containing $g_{m^{\prime}, l^{\prime}}, g_{m^{\prime}, l^{\prime}} \in \mathcal{O}\left(g_{m, l}\right)$, otherwise set $\mathcal{F} Y[m, l]=0$ if $I^{\text {svd }}\left(\mathcal{F} X_{\gamma}\right)<T$ for all $\gamma$ containing $g_{m^{\prime}, l^{\prime}}, g_{m^{\prime}, l^{\prime}} \in \mathcal{O}\left(g_{m, l}\right)$.

(C6) Let $Y$ be the inversion of $\mathcal{F} Y$. Set $\tilde{F}=\tilde{F}+Y$ and $X=X-Y$.

(C7) Choose a paramenter $\epsilon>0$, if $|Y|>\epsilon$ go to step (C2).

Note that the details of the implementation (C1)-(C7) are in line with the general strategy of matching pursuit. The window length $q$ in step (C2) could change from one iteration to the next to 'extract' possible structure belonging to the underlining signal at several different scales. In the experiments performed in the following section we alternate between two window sizes $q_{1}$ and $q_{2}$.
The attenuation introduced in (C5) has some additional ad hoc parameters in the definition of the neighborhoods in (10) and in the choice of the attenuation parameter $\alpha$. By the double process of increasing the number of nonzero coefficients chosen at each step and decreasing their contribution we are allowing more information to be taken at each iteration of the projection pursuit algorithm, but in a slow learning framework that in principle (and in practice as we found out) should increase the sharpness of the distinct features of the estimate, on the general issue of attenuated learning processes see the discussion in $[\mathrm{HTF}]$ chapter 10 . Note that the attenuation coefficient leads to improved results only when it is part of a recursive algorithm, otherwise it gives only a rescaled version of the estimate.

One drawback of the algorithm we described is the need to choose several parameters: we choose a dictionary of analysis $\mathcal{D}$, a collection of discrete paths $C_{p}$, the embedding parameters $\tau$ (time delay) and $d$ (embedding dimension), and the learning parameters $T$ (threshold level), $\alpha$ (attenuation coefficient) and $\epsilon$. Again we stress that all such choices are context dependent, and are the price to pay to have an estimator that is relatively intensity independent and applicable to wide classes of noise distributions.

The choice of $\mathcal{D}$ is dependent on the type of signals we analyze and we do not see a serious need to make such choice automatic.

Since we analyze speech signals, we choose the dictionary to be the set of atoms of the windowed Fourier frames; the algorithm is not very sensitive to the choice of the length $q$ of the window in the Fourier frame, while the use of several windows is found to be always beneficial.

The choice of $C_{p}$ is also dependent on the type of signals analyzed, speech signals have specific frequencies that change in time, so a set of paths parallel to the time axis was natural in this case. Let us explore now the relation of parameters associated with $C_{p}$, embedding parameters $\tau$ and $d$ and threshold $T$. Recall that for the collection $C_{p}$ we have as parameters the time and frequency sampling rates $\bar{l}$ and $\bar{m}$ and the length $p$ of the paths. The frequency sampling rates $\bar{l}$ and $\bar{m}$ are necessary only to speed up the algorithm, ideally we would like a dense sampling. Same considerations apply to the 'thickening' of the paths in (10), we basically try to speed up the algorithm by collecting more data at each iteration.

So the only essential parameters are the path length $p$, the embedding parameters and the threshold $T$

Essentially we want to set these parameters so that the number of paths that have index $I^{\text {svd }}>T$ is sizeable for a training set of speech signals and marginal for the white noise time series of interest.

Our experience is that such choice is possible and robust, we gave a simple rule to find the threshold $T$ in step (A) in the previous section given a choice of $(p, \tau, d)$.

A learning algorithm could be built to find $T$, the paths' length $p$, and the embedding parameters, namely let $\bar{Q}_{S}(x)$ be the mean of the functions $Q_{S_{i}}(x)$ for a training set of speech signals $S_{i}$ and $\bar{Q}_{W}(x)$ be the mean of the functions 
$Q_{W_{i}}(x)$ for a set of white noise time sieries $W_{i}$

We can first find $d, \tau$ and $p$ such that the distance of the functions $\bar{Q}_{W}(x)$ and $\bar{Q}_{S}(x)$ is maximum in the $L^{2}$ norm. After finding these parameters, we can find a value of $T$ such that $T$ is the smallest positive number with $\bar{Q}_{S}(T)$ one order of magnitude larger than $\bar{Q}_{W}(T)$, as we did in (A) in the previous section, to make our algorithm automatically applicable to data sets of interest different from speech signals it will be necessary to formalize this optimization procedure.

Finally the choice of $\alpha$ and $\epsilon$ is completely practical in nature, ideally we want $\alpha$ and $\epsilon$ as close to zero as possible, but, to avoid making the algorithm unreasonably slow, we must set values that are found to give good quality reconstructions on some training set of speech signals while they require a number of iterations of the algorithm that is compatible with the computing and time requirements of the specific problem. For longer time series, as the ones in the next section, we segment the data in several shorter pieces, and we iterate the algorithm a fixed number of times $k$ rather than using $\epsilon$ in $(\mathrm{C} 7)$ to decide the number of iterations.

Note:The algorithm described in this paper is being patented, with provisional patent application number 60/562,534 filed on April 16, 2004.

\section{DENOISING}

In this section we explore the quality of the attenuated embedding threshold as implemented in the windowed Fourier frame and with our class of paths $\mathcal{C}_{p}$. We apply the algorithm to 10 speech signals from the TIMIT database contaminated by different types of white noise with several intensity levels. We show that the attenuated embedding threshold estimator performs well for all white noise contaminations we consider. The delay along the paths is chosen as $\tau=4$, the length of the paths is $p=2^{8}$ and the window length of the windowed Fourier transform alternates between $q=100$ and $q=25$ (to detect both features with good time localization and those with good frequency localization), the embedding dimension $d=4$. For these parameters and for the set of speech signals that we used as training, we have $T \approx 26.8$ when $q=100$ and $T \approx 27.4$ when $q=25$ using the procedure (A) of section III.

The sampling interval of the paths in the frequency direction is $S_{\bar{m}}=3$ and along the time direction is $S_{\bar{l}}=p / 2 \mathrm{We}$ select $\alpha=0.1$, as small values of $\alpha$ seem to work best (see discussion in the previous section). The algorithm is applied to short consecutive speech segments to reduce the computational cost of computing the windowed Fourier transform on very long time series, therefore, to keep the running time uniformly constant for all such segments, we decided to iterate the algorithm (C1)-(C6) a fixed number of times (say 6 times) instead of choosing a parameter $\epsilon$ in (C7).

As we already said, the window size $q$ in (C2) alternates between $q=100$ and $q=25$. It is moreover important to note that the attenuated embedding threshold is able to extract only a small fraction of the total energy of the signal
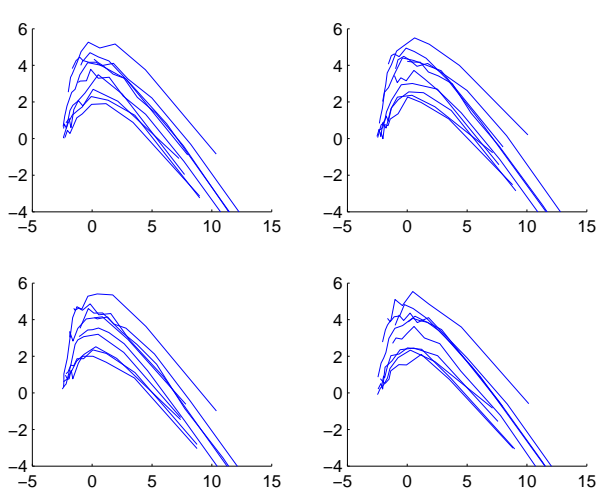

Fig. 3. Scaled SNR gain in decibel of the attenuated embedding estimates plotted against the scaled SNR of the corresponding measurements. From top left in clockwise order we consider the case of: a)Gaussian white noise; b) uniform noise; c)Tukey white noise; d)discrete bimodal distribution .

$f$, exactly because of the attenuation process, therefore the Signal-to-Noise Ratio $(S N R)$ computations are done on scaled measurements $X$, estimates $\tilde{F}$, and signals $F$ set to be all of norm 1. We call such estimations scaled $S N R$, and we explicitely write, for a given signal $F$ and estimation $Z$,

$$
S N R_{s}(Z)=10 \log _{10} \frac{1}{E(|F /| F|-Z /| Z \mid)}
$$

We then compute $S N R_{s}(X)$ and $S N R_{s}(\tilde{F})$ by approximating the expected values $E(|F /| F|-X /| X \mid)$ and $E(|F /| F|-\tilde{F} /| \tilde{F} \mid)$ with an average over several realizations for each white noise contamination.

In Figure 3 we show the gains of the scales SNR of the reconstructions (with the attenuated embedding threshold estimator) plotted against the corresponding scaled SNR of the measurements. Each curve correspond to one of 10 speech signals of approximately one second used to test the algorithm. From top left in clockwise direction we have measuremets contaminated by random processes with pdfs 1) to 4) as defined in section III and with several choices of variance. Note that the overall shape of the scaled SNR gain is similar for all distributions (notwithstanding that the discrete plots do not have exactly the same domain). The maximum gain seems to happen for measurements with scaled SNR around 1 decibel. Note that the right tail of the SNR gains takes often negative values; this is due to the attenuation effect of the estimator that is pronunced for the high intensity speech features, but it is not necessarily indicative of worse perceptual quality with respect to the measurements, some of the figures in the following will clarify this point.

In the first case of Gaussian white noise, we compared our algorithm to the block thresholding algorithm described in [CS], we used the matlab code implemented by [ABS], made available at www.jstatsoft.org/v06/i06/codes/ as a part of their thourogh comparison of denoising methods. As the block thresholding estimator is implemented in a symmlet wavelet basis that is not well adapted to the structure of 


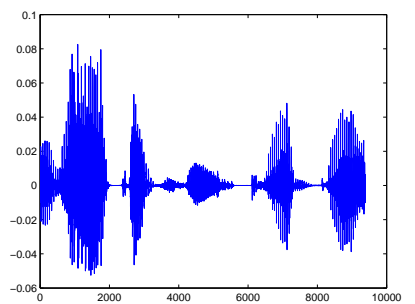

Fig. 4. Signal 'SPEECH10' scaled to have norm 1.

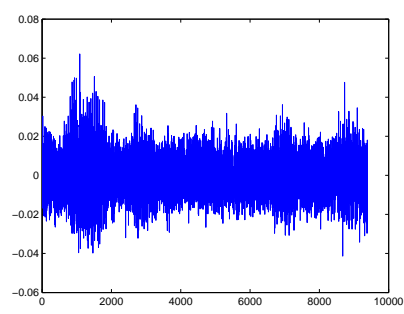

Fig. 5. Noisy scaled measurement of SPEECH10 with Gaussian white noise and scaled SNR of about $1 \mathrm{db}$.

speech signals, a more compelling comparison would require the development of an embedding threshold estimator in a wavelet basis, we plan to do so in a future work. In Figure 10 we show the scaled SNR gain for all tested speech signals using the block threshold estimator (right plot) and attenuated embedding estimator (left plot). In Figure 4 we show one original speech signal, Figure 5 shows the measurement in the presence of Gaussian noise corresponding to the 'peak' of the $S N R_{s}$ gain curve (measurement $S N R_{s} \approx 1$ ), Figure 6 shows the corresponding reconstruction with attenuated embedding threshold estimator. Similarly Figure 7 shows another speech signal, while Figure 8 shows the measurement with Tukey noise corresponding to the 'peak' of the Tukey noise $S N R_{s}$ gain curve (measurement $S N R_{s} \approx 1$ ), Figure 9 shows the reconstruction. In both cases the perceptual quality is better than the noisy measurements, which is not necessarily the case for estimators in general.

Note moreover that even though $T$ was found using only Gaussian white noise as the training distribution, none of the parameters of the algorithm were changed as we went from Gaussian white noise contaminations to more general white noise processes, and yet the $S N R_{s}$ gain was similar, it must

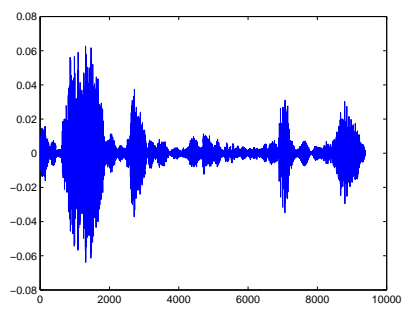

Fig. 6. Attenuated embedding estimate of SPEECH10 from the measurement in Figure 6, scaled to have norm 1.

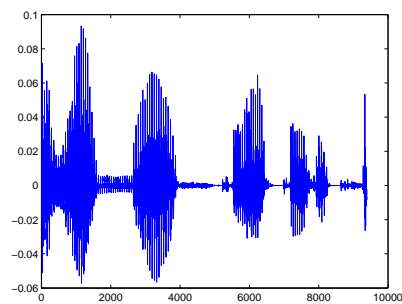

Fig. 7. Signal 'SPEECH5' scaled to have norm 1.

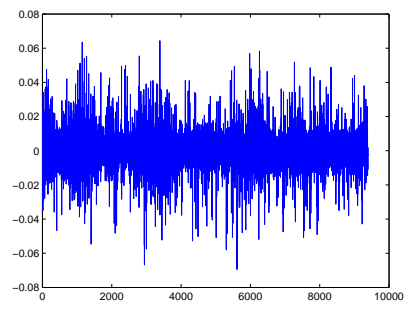

Fig. 8. Noisy measurement of SPEECH5 with Tukey white noise and scaled SNR of about $1 \mathrm{db}$.

be noted though that the estimates for bimodal and uniform noise were not intelligible at the peak of the $S N R_{s}$ gain curve (just as the measurements were not).

Since the performance of the embedding estimator is not well represented by the scaled SNR for low intensity noise (measurements appear to be better than the estimates), in Figures 10 to 21 we show two more instances of speech signals contaminated by lower variance Tukey noise, Gaussian noise and discrete bimodal noise (uniform noise leads to reconstructions very similar to the discrete bimodal distribution), for one case of low Gaussian white noise we show a block thresholding estimate, note how the low intensity details are lost, this inability to preserve low intensity details worsens when higher variance noise is added, but then again, it must be tempered by the fact that a standard wavelet basis is not well adapted to the structure of speech signals.

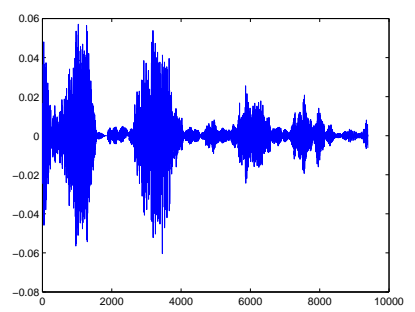

Fig. 9. Attenuated embedding estimate of SPEECH5 from the measurement in Figure 9, scaled to have norm 1. 


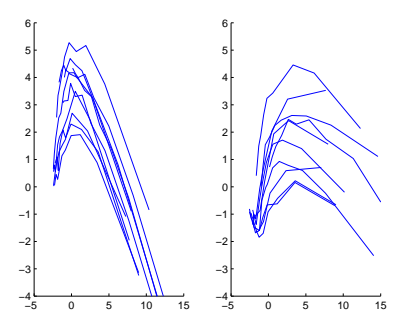

Fig. 10. $S N R_{s}$ gain for the estimates of 10 speech signals and Gaussian additive noise using: the block thresholding estimator of [CS](right), the embedding threshold estimator(left).

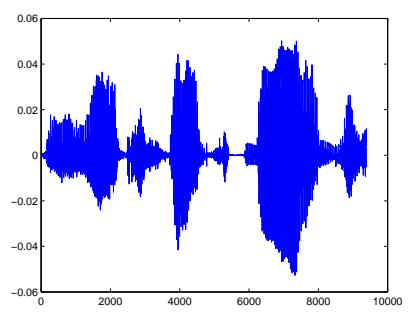

Fig. 11. Signal 'SPEECH2' scaled to have norm 1.

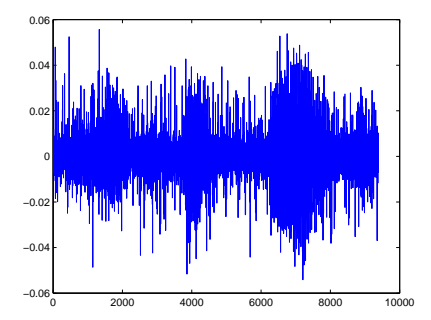

Fig. 12. Noisy measurement of SPEECH2 with Tukey white noise and scaled SNR of about $4.4 \mathrm{db}$.

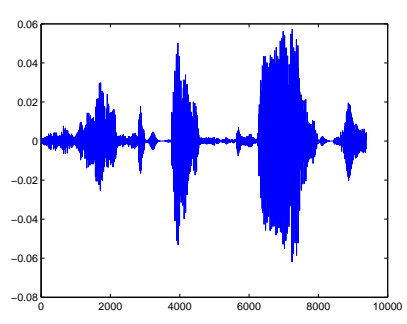

Fig. 13. Attenuated embedding estimate of SPEECH2 from the measurement in Figure 12, scaled to have norm $1, S N R_{s}$ is $\approx 8.1 \mathrm{db}$.

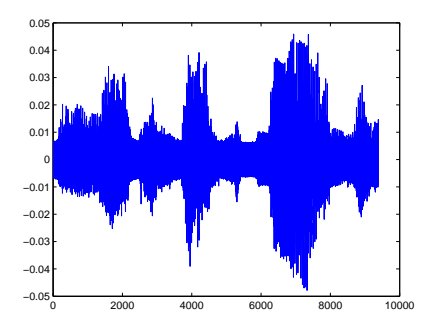

Fig. 14. Noisy measurement of SPEECH2 with bimodal white noise and scaled SNR of about $4.5 \mathrm{db}$.

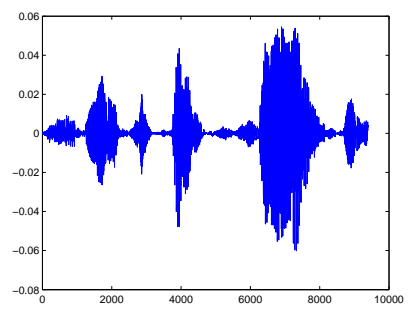

Fig. 15. Attenuated embedding estimate of SPEECH2 from the measurement in Figure 14 , scaled to have norm $1, S N R_{s}$ is $\approx 8.1 \mathrm{db}$.

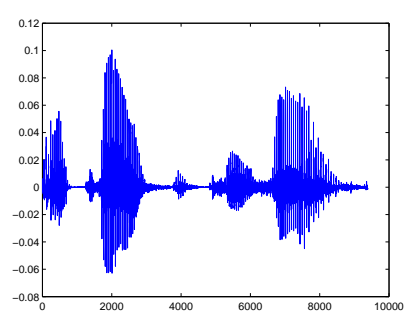

Fig. 16. Signal 'SPEECH7' scaled to have norm 1.

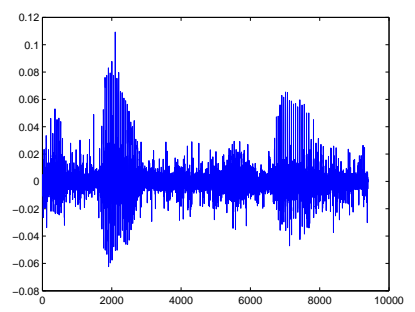

Fig. 17. Noisy measurement of SPEECH7 with Tukey white noise and scaled SNR of about $7.3 \mathrm{db}$ 


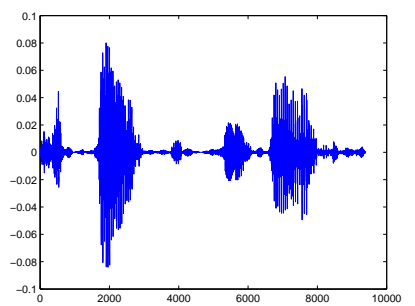

Fig. 18. Attenuated embedding estimate of SPEECH7 from the measurement in Figure 17, scaled to have norm $1, S N R_{s}$ is $\approx 6$.

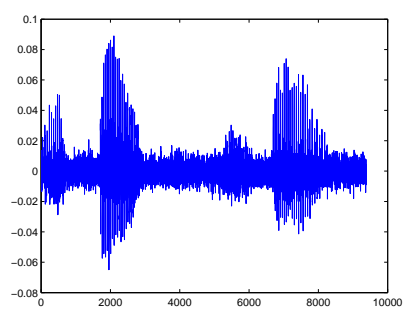

Fig. 19. Noisy measurement of SPEECH7 with Gaussian white noise and scaled SNR of about $11.1 \mathrm{db}$.

Data files for the signal, measurement and reconstructions used to compute the quantities in all the figures are available upon request for direct evaluation of the perceptual quality.

\section{FuRTher DeVElopments}

Given that the embedding threshold ideas were implemented with the specific goal of denoising speech signals, it may be worth emphasizing that in principle the construction of classes of paths can be applied to other dictionaries well adapted to other classes of signals, more paricularly, let $\mathcal{D}=\left\{g_{1}, \ldots, g_{P}\right\}$ be a generic frame dictionary of $P>N$ elements so that $X=\sum_{m=1}^{P} X_{\mathcal{D}}[m] \tilde{g}_{m}, X_{\mathcal{D}}[m]=<X, g_{m}>$, where $\tilde{g}_{m}$ are dual frame vectors (see [M] ch.5). Given such a general representation for $X$, let $\mathcal{C}_{p}=\left\{\gamma_{1}, \ldots, \gamma_{Q}\right\}, Q>P$, be a collection of ordered subsets of $\mathcal{D}$ of length $p$, that is, $\gamma_{i}=\left\{g_{i_{1}}, \ldots, g_{i_{p}}\right\}$, so that $\bigcup \gamma_{i}=\mathcal{D}$ and the cardinality of the set $\left\{\gamma_{i}\right.$ such that $\left.g_{j} \in \gamma_{i}\right\}$ is constant for every $j=0, \ldots, P-1$ (this ensures that the discrete covering of the frame atoms is locally uniform). Note that $\mathcal{C}_{p}$ needs not be the entire set of ordered subsets of $\mathcal{D}$. We call each $\gamma_{i}$ a 'path' in $\mathcal{D}$ for reasons that will be clear in the following. Let $X_{\gamma_{i}}=\left\{X_{\mathcal{D}}[m]=<X, g_{m}>, g_{m} \in \gamma_{i}\right\}$ be an ordered

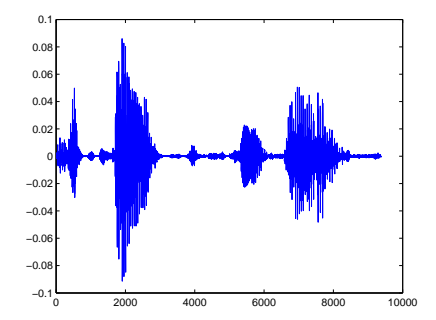

Fig. 20. Attenuated embedding estimate of SPEECH7 from the measurement in Figure 19, scaled to have norm $1, S N R_{s}$ is $\approx 7.7$.

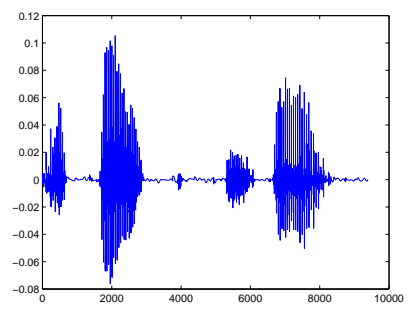

Fig. 21. Block thresholding estimate of SPEECH7 from the measurement in Figure 19 , scaled to have norm $1, S N R_{s}$ is $\approx 7.6$, note low intensity details are removed by the estimator.

collection of coefficients of $X$ in the dictionary $\mathcal{D}$.

Then a a semi-local estimator in $\mathcal{D}$ can be defined as:

$$
\tilde{F}=\sum_{m=0}^{P-1} d_{I, T}\left(X_{\mathcal{D}}[m]\right) \tilde{g}_{m}
$$

where $d_{I, T}\left(X_{\mathcal{D}}[m]\right)=X_{\mathcal{D}}[m]$ if $I\left(X_{\gamma}\right) \geq T$ for some $\gamma$ containing $m$, and $d_{I, T}\left(X_{\mathcal{D}}[m]\right)=0$ if $I\left(X_{\gamma}\right)<T$ for all $\gamma$ containing $m$.

The construction of significant sets of paths $\mathcal{C}_{p}$ will depend from the application, we are currently exploring even the possibility of using random walks along the atoms of the dictionary $\mathcal{D}$. In any case, after $C_{p}$ is selected, our specifc choice of index $I^{\text {svd }}$ can be used and the attenuated embedding estimator can certainly be applied and tested, soft threshold embedding estimators are an interesting open possibility as well.

\section{REFERENCES}

[ABS] A. Antoniadis, J. Bigot, T. Sapatinas, Wavelet Estimators in Nonparametric Regression: A Comparative Simulation Study, 2001, available http://www.jstatsoft.org/v06/i06/

[ASY] K. T. Alligood, T. D. Sauer, J. A. Yorke, Chaos. An introduction to Dynamical systems, Springer, 1996.

[C] T. Cai, Adaptive wavelet estimation: a block thresholding and oracle inequality approach. The Annals of Statistics 27 (1999), 898-924.

[CL] T. Cai, M. Low, Nonparametric function estimation over shrinking neighborhoods: Superefficiency and adaptation. The Annals of Statistics 33 (2005)., in press.

[CS] T. Cai, B. W. Silverman, Incorporating information on neighboring coefficients into wavelet estimation, Sankhya 63 (2001), 127-148.

[DMA]G. Davis, S. Mallat and M. Avelaneda, Adaptive Greedy Approximations, Jour. of Constructive Approximation, vol. 13, No. 1, pp. 57-98, 1997

[DJ] D. Donoho, I. Johnstone, Minimax estimation via wavelet shrinkage. Annals of Statistics26 : 879921,1998 . 
[ELPTS. Efromovich, J. Lakey, M.C. Pereyra, N. Tymes, Data-driven and optimal denoising of a signal and recovery of its derivative using multiwavelets, IEEE transaction on Signal Processing, 52 (2004) ,628635.

[KS] H. Kantz, TSchreiber ,Nonlinear Time Series Analysis, Cambridge University Press, 2003.

[HTF] T. Hastie, R. Tibshirani, J. Friedman, The Elements of Statistical Learning, Springer, 2001.

[LE] E. N. Lorenz, K. A. Emanuel, Optimal Sites for Supplementary Weather Observations: Simulation with a Small Model. Journal of the Atmospheric Sciences 55, 3 (1998), 399414.

[M] S. Mallat, A Wavelet Tour of Signal Processing, Academic Press, 1998.

[Me] A. Mees (Ed.), Nonlinear Dynamics and Statistics, Birkhauser, Boston, 2001.

[S] T. Strohmer, Numerical Algorithms for Discrete Gabor Expansions, in Gabor Analysis and Algorithms. Theory and Applications, H. G. Feichtinger, T. Strohmer editors. Birkhauser, 1998.

[SYC] T. Sauer, J. A. Yorke, M. Casdagli, Embedology, Journal of Statistical Physics,65 (1991), 579-616. 